\title{
Estimación de la incertidumbre global de un procedimiento para la determinación de humedad por gravimetría en platos preparados (Raciones individuales de combate)
}

\author{
Pérez Grana R. ${ }^{1}$, Zamora Benito A. ${ }^{2}$, López Tomás LA. ${ }^{1}$, Calonge Jiménez MR. ${ }^{2}$, Macho Martínez \\ MA. ${ }^{3}$, Rodríguez-Marín Roy Jl. ${ }^{1}$
}

Sanid. mil. 2012; 68 (2): 73-78; ISSN: 1887-8571

RESUMEN

Objetivos: Realizar una estimación de la incertidumbre de forma global para la determinación de humedad en platos preparados por gravimetría aprovechando los datos obtenidos en el proceso de validación. Material y métodos: Se analiza, mediante un diseño anidado, un material de referencia certificado (MRC) con un contenido de humedad certificado de 61,8 $\pm 0,7 \mathrm{~g} / 100 \mathrm{~g}$. La estimación de la incertidumbre combinada, se halla aplicando la ley de propagación de la incertidumbre. La incertidumbre expandida se obtiene aplicando un factor de cobertura $\mathrm{K}=2$ (nivel de confianza del 95\%). Mediante la aproximación SUMU se añade el sesgo a la incertidumbre expandida. Resultados: El límite de repetibilidad y reproductibilidad, para un nivel de confianza del 95\%, es igual a $0,58 \%$ y $1,21 \%$, respectivamente. Se obtienen los siguientes valores de incertidumbre: duplicados $(0,12 \%)$, precisión $(0,44 \%)$, trazabilidad/ sesgo $(0,39 \%)$, incertidumbre combinada $(0,60 \%)$ e incertidumbre expandida corregida (1,38 \%). Conclusiones: El uso de un diseño anidado (analista, instrumento, día, replicado), permite variar los factores de una forma ordenada, entre cada serie, y estimar la precisión intermedia debida a varios factores. El cálculo de la incertidumbre de forma global supone una ventaja en cuanto a simplicidad, especialmente para laboratorios con pocos recursos humanos y materiales. La inclusión del sesgo experimental no significativo como un componente de la incertidumbre tiende a evitar la infraestimación de la incertidumbre de los resultados.

PALABRAS CLAVE. Incertidumbre global. Humedad. Platos preparados.

Nutritional analysis in ready meals (individual combat rations), Calculation of Uncertainty global estimated in a procedure for determination of moisture by gravimetric technique.

SUMMARY

Objectives: The aim of this article was to estimate the uncertainty total for the determination of moisture in ready meals by gravimetric using data obtained in the validation process. Material and methods: Samples were analyzed by gravimetric method in certified reference material (CRM) (moisture certificate content: $61.8 \pm 0.7$ a $100 \mathrm{~g}$.) in nested design. The combined uncertainty was estimated by applying the law of propagation of uncertainty. The expanded uncertainty was obtained by applying a coverage factor $\mathrm{K}=$ 2 (confidence level 95\%). Bias was added to the expanded uncertainty by the approximation SUMU. Results: The repeatability and reproducibility limit for a confidence level of $95 \%$ is equal to $0.58 \%$ and $1.21 \%$ respectively. You get the following values of uncertainty: duplicate $(0.12 \%)$, precision $(0.44 \%)$, traceability / bias $(0.39 \%)$ combined uncertainty $(0.60 \%)$ and revised expanded uncertainty $(1.38 \%)$. Conclusions: Using a nested design (analyst, instrument, day, replicated), can vary the factors in an orderly manner, between each series, and intermediate precision estimate due to several factors. Calculation of a global uncertainty is an advantage in terms of simplicity, especially for laboratories with limited human and material resources. Inclusion of experimental bias not significant as a component of uncertainty tends to avoid underestimation of the uncertainty of the results.

KEYWORDS: Global uncertainty, moisture, ready meals.

\section{INTRODUCCIÓN}

En un mercado global como el actual existe una considerable demanda por la comparabilidad de los resultados analíticos, ya

\footnotetext{
${ }^{1}$ Tcol. Veterinario.

2 Cte. Veterinario.

${ }^{3}$ Cap. Veterinario.

Centro Militar de Veterinaria de la Defensa. Servicio de Bromatología y Seguridad Alimentaria. Madrid. España.
}

Dirección para correspondencia: Roberto Pérez Grana. Servicio de Bromatología y Seguridad Alimentaria. Centro Militar de Veterinaria de la Defensa. Madrid. C/ Darío Gazapo, 3. 28024 Madrid. Tfno: 915122515.rpergra@oc.mde.es

Recibido: 17 de marzo de 2011

Aceptado: 15 de diciembre de 2011 que de forma habitual se usan como información para tomar decisiones acerca de la conformidad con un valor límite especificado en la legislación o en una especificación técnica. En el caso de platos preparados, entre los que se incluyen las raciones individuales de combate, esta información se utiliza para conocer sus características nutricionales y/o si se cumplen unas especificaciones técnicas. Por consiguiente, para comparar los resultados de diferentes laboratorios deben ser expresados como resultado \pm incertidumbre. En este sentido, la norma ISO/IEC $17025^{1}$ indica que los resultados analíticos deben ir acompañados de su incertidumbre y la validación debe ser lo extensa que sea necesario para atender a las necesidades de aplicación. La validación consiste en comprobar que el resultado obtenido representa el contenido real del analito en las muestras de rutina, y 


\section{R.Pérez Grana, et al.}

supone investigar de forma experimental y matemática, teniendo en cuenta el intervalo de concentraciones y matrices, las posibles fuentes de error de cada una de las etapas y que el método responde a su finalidad ${ }^{2}$. Asimismo, validar un método consiste en su adecuación a unos criterios establecidos por el usuario de los resultados (cliente externo, servicio veterinario). Con frecuencia sucede que el usuario no está capacitado para definir un determinado nivel de precisión o trazabilidad y es el responsable del laboratorio el que fija tales parámetros de calidad. Esta noción amplia de lo que implica la validación concuerda con el concepto de adecuación a la finalidad o requerimientos perseguidos ("Fitness for purpose") y es lo que debería llegar a declarar el método de validación ${ }^{3}$. Este término es definido por la IUPAC ${ }^{4}$ como el nivel alcanzado por los datos producidos por un proceso de medida que permite al usuario tomar decisiones técnica y administrativamente correctas para un proceso determinado.

Dentro de los parámetros de calidad fundamentales para validar un método, tenemos: la verificación de la exactitud o trazabilidad del método analítico y el cálculo del valor numérico de la incertidumbre.

La trazabilidad se define según el Vocabulario Internacional de Metrología ${ }^{5}$ como la propiedad del resultado de una medida que le permite relacionarlo con referencias determinadas, generalmente nacionales o internacionales, a través de una cadena ininterrumpida de comparaciones todas ellas de incertidumbres conocidas. Asegurar la trazabilidad de un resultado implica decir que el resultado es comparable al proporcionado por la referencia utilizada y que, por tanto, es exacto. Y un resultado es exacto si simultáneamente es veraz o trazable (se encuentra libre de errores sistemáticos) y preciso (los errores aleatorios son aceptables)

Por ello, el concepto de trazabilidad es muy similar al concepto de exactitud y es una forma de que el usuario tenga confianza en los resultados analíticos. Sin embargo, en el análisis químico con respecto a las medidas físicas, es muy difícil llegar a través de una cadena ininterrumpida de comparaciones a la unidad de medida del sistema internacional (SI) básica ${ }^{6}$, el mol, ya que en la mayoría de los procedimientos analíticos no es posible asegurar la trazabilidad de cada una de las etapas del procedimiento. La alternativa para los laboratorios consiste en trazar globalmente el procedimiento analítico a una referencia: métodos definitivos, materiales de referencia certificados, métodos de referencia, ejercicios interlaboratorio, muestras adicionadas ${ }^{7} y$ otras. Los métodos definitivos tienen el más alto nivel jerárquico de trazabilidad; pero en la mayor parte de las veces es imposible utilizar métodos definitivos para verificar la trazabilidad. No obstante, el uso de referencias adecuadas es suficiente para poder tener el grado suficiente de comparabilidad entre resultados sin tener que llegar a la unidad de medidas del SI ${ }^{8}$.

Demostrar documentalmente que los resultados son exactos o trazables tiene la ventaja de proporcionar seguridad al laboratorio que emite los resultados ante una reclamación por un informe incorrecto, con posibles consecuencias económicas por retirada de lotes, y/o legales.

Según la norma ISO 3534-19 , la exactitud se define como el grado de concordancia entre el resultado de un ensayo y el valor de referencia aceptado. Por tanto, además de considerar los erro- res sistemáticos (determinados), el término exactitud considera también los errores aleatorios (indeterminados).

Por otro lado, la precisión se define como la dispersión de los valores obtenidos alrededor de un valor medio. La precisión muy a menudo varía con la concentración de analito ${ }^{10}$, permite evaluar los errores aleatorios y nos indica una dispersión de los datos. La precisión se puede obtener en condiciones de repetibilidad y en condiciones de reproductibilidad. Entre estas dos, podemos encontrar diferentes condiciones intermedias de precisión, por ejemplo, diversas réplicas en diversos días cambiando instrumento y analista; pero dentro de un mismo laboratorio, que son las condiciones que tradicionalmente se conocen como reproducibilidad.

La incertidumbre se define como una estimación unida al resultado de un ensayo que caracteriza el rango de valores dentro de los cuales se afirma que está el valor verdadero. La diferencia más importante entre incertidumbre y precisión se encuentra en el hecho de que el concepto de incertidumbre está íntimamente relacionado con el concepto de trazabilidad.

Aunque existen varias aproximaciones para el cálculo de la incertidumbre, se tiende a aprovechar la información obtenida en el proceso de validación del método, agrupando términos siempre que sea posible, y calcular la incertidumbre de una forma más global ${ }^{11,12}$.

\section{MATERIAL Y MÉTODOS}

Las muestras de platos preparados se trituran durante periodos breves, para evitar el calentamiento ${ }^{13}$, hasta obtener un tamaño de partícula $\leq 1 \mathrm{~mm}^{14}$. Para la determinación de humedad en platos preparados por gravimetría se toma como base la norma ISO para el análisis de humedad en carne y productos cárnicos ${ }^{15}$.

La validación se realiza dentro del laboratorio o validación in-house. En la verificación de la exactitud o trazabilidad del método se utiliza un material de referencia certificado ERMBB501 LGC (Carne procesada) con un contenido de humedad certificado de $61,8 \pm 0,7 \mathrm{~g} / 100 \mathrm{~g}$.

Siguiendo las recomendaciones de la ISO $5725-3^{16}$, el material de referencia certificado se analiza estableciendo un diseño anidado que permite variar varios factores (analista, instrumento, día, replicado) de una forma ordenada. La pesada se realiza en días diferentes para evitar infraestimar el efecto del día sobre la variabilidad de los resultados.

El cálculo de la repetibilidad se realiza a partir de la expresión siguiente:

$$
\mathrm{S}_{\mathrm{r}}=\sqrt{S_{1}^{2}+S_{2}^{2}+S_{3}^{2}+\ldots S_{n}^{2} / n}
$$

Donde Sr es la estimación de la desviación estándar de la repetibilidad del procedimiento analítico al analizar el material de referencia certificado (MRC) y $S_{n}^{2}$ es la desviación estándar entre replicados $\mathrm{y} \mathrm{n}$ es el número de duplicados. Y los límites de repetibilidad y reproductibilidad se calculan según las expresiones:

$$
1,96 \sqrt{2 S_{r}^{2}} \text { y } 1,96 \sqrt{2 S_{r}^{2}}
$$




\section{Estimación de la incertidumbre global de un procedimiento para la determinación de humedad...}

Donde Sr es la estimación de la desviación estándar de la repetibilidad del procedimiento analítico y $\mathrm{S}_{\mathrm{I}}$ es la estimación de la desviación estándar intermedia del procedimiento analítico.

Se asume que el error sistemático es el mismo en el intervalo de aplicación del método, que se establece entre el $40 \%$ y 95\% de humedad.

Para el estudio del sesgo, y en consecuencia, verificar la trazabilidad del procedimiento se usa el estadístico t, de dos colas, para determinar si el valor medio del contenido de humedad es significativamente diferente del valor certificado, según la expresión:

$$
\mathrm{t}_{\exp }=\frac{\left|V_{n}-V_{m}\right| \sqrt{n}}{S}
$$

Donde Vn es el valor nominal del material de referencia certificado, $\mathrm{Vm}$ es el valor medio de los análisis del material de referencia certificado, $\mathrm{S}$ es la desviación estándar de los resultados y n, el número de observaciones. Para el cálculo de $t_{\text {exp }}$ se pueden emplear grados de libertad (n-1), o grados de libertad efectivos, que se calculan utilizando la aproximación de WelchSattherthwaite.

A partir de las muestras remitidas por las UCO,s se analizan un total de 10 muestras de platos preparados por duplicado, que representan la variabilidad de las muestras de rutina ${ }^{17}$ en cuanto a matrices y concentraciones. La incertidumbre de las muestras de duplicados se calcula utilizando la expresión:

$$
\mathrm{u}_{\text {Duplicados }}=\mathrm{S} / \sqrt{n}
$$

Donde $\mathrm{S}$ es la estimación de la desviación estándar de las diferencias entre duplicados y n es el duplicado, que se sustituye por 2. Siguiendo las recomendaciones indicadas en la bibliografía $^{18}$, se calcula la incertidumbre de la precisión y de la trazabilidad/sesgo. La incertidumbre de la precisión del procedimiento se calcula según la expresión:

$$
\mathrm{u}_{\text {Precisión }}=\frac{S_{I}}{\sqrt{n}}(3)
$$

Donde $\mathrm{S}_{\mathrm{I}}$ es la estimación de la desviación estándar intermedia del procedimiento y n, el número de veces que se analiza la muestra de rutina, normalmente $\mathrm{n}$ igual a 1 .

Esta incertidumbre se calcula a partir de la precisión intermedia del método. Es debida a la variación experimental obtenida cuando se aplica el procedimiento analítico a muestras futuras. Este término está relacionado con la variabilidad de los resultados analíticos debida a los errores aleatorios derivados del procedimiento analítico y de las condiciones mediante las cuales se realiza el análisis (analista, instrumento, día).

El cálculo de la incertidumbre de la trazabilidad/sesgo se obtiene según la expresión:

$$
\mathrm{u}_{\text {Trazabilidad/sesgo }}=\sqrt{u_{r e f}^{2}+\left(\frac{s_{I}}{\sqrt{n}}\right)^{2}}
$$

Donde $\mathrm{u}_{\mathrm{ref}}$ es la incertidumbre estándar del material certificado de referencia y n es el número de series y $\mathrm{S}_{\mathrm{I}}$, la estimación de la desviación estándar de la precisión intermedia del procedimiento. Esta expresión corresponde a la incertidumbre del sesgo, puesto que éste tiene una incertidumbre asociada, teniendo en cuenta que no se tiene una seguridad total de que los resultados resulten trazables.

Aplicando la ley de propagación de la incertidumbre ${ }^{19}$ tenemos la expresión básica que estima la incertidumbre:

$$
\mathrm{u}\left[\mathrm{y}\left(\mathrm{x}_{1}, \mathrm{x}_{2}, \ldots\right)\right]=\sqrt{\sum_{i=1, n} c_{i}{ }^{2} u\left(x_{i}\right)^{2}}
$$

Donde $y\left(x_{1}, x_{2}, \ldots . . x_{n}\right)$ es una función de varias variables independientes $\mathrm{x}_{1}, \mathrm{x}_{2}, \ldots, \mathrm{y} \mathrm{c}_{\mathrm{i}}$ es el coeficiente de sensibilidad evaluado como $\mathrm{c}_{\mathrm{i}}=\delta \mathrm{y} / \delta \mathrm{x}_{\mathrm{i}}$, que es la derivada parcial de $\mathrm{y}$ con respecto $\mathrm{a}_{\mathrm{i}}, \mathrm{u}\left(\mathrm{x}_{\mathrm{i}}\right)$ y $\mathrm{u}(\mathrm{y})$ son la incertidumbre estándar y la incertidumbre estándar combinada.

Aplicando la expresión anterior, el cálculo de la incertidumbre combinada de la humedad (\%) se obtiene según la expresión:

$$
\mathrm{u}_{\mathrm{c}(\text { Humedad })}=\sqrt{u^{2} \text { Pr ecisión }+u^{2} \text { Traz/Sesgo }+u^{2} \text { Duplicados }}
$$

Por tanto, la incertidumbre combinada se calcula de forma global agrupando términos correspondientes a la validación del método (precisión y trazabilidad) e incertidumbre de duplicados.

La incertidumbre expandida $U$, se obtiene multiplicando la incertidumbre combinada estándar por un factor de cobertura $\mathrm{k}$, basado en una distribución normal. Habitualmente se utiliza un factor $\mathrm{k}=2$, asimilable a un nivel de significación $\alpha$ del $5 \%{ }^{20}$.

La incertidumbre total expandida se obtiene mediante la expresión:

$\mathrm{U}=\mathrm{k} \mathrm{u}_{\mathrm{c}(\text { Humedad) }}, \mathrm{K}=2$ (nivel de confianza del 95\%).

$\mathrm{U}_{\text {(Humedad) }}=2 \times \mathrm{u}_{\mathrm{c} \text { (Humedad) }}(6)$

$\mathrm{U}_{\text {Muestra }}=\mathrm{c} \times \mathrm{U}_{\text {(Humedad) }}$

Donde c es la concentración de humedad (\%) hallada en la muestra de rutina. Mediante la aproximación $\mathrm{SUMU}^{21}$ se añade el sesgo a la incertidumbre expandida y es equivalente a corregir el resultado $\mathrm{U}_{+}=\mathrm{U}+$ sesgo.

\section{RESULTADOS}

A partir de los análisis del MRC, siguiendo un diseño anidado, se obtienen los siguientes resultados de cada serie (expresados en $\%$ de humedad) en diferentes días: día $1(61,29 \%)$, día 2 $(61,29 \%)$, día $3(61,48 \%)$, día $4(61,82 \%)$, día $5(62,46 \%)$, día 6 $(62,09 \%)$ y día $7(61,46 \%)$. No se hallan resultados discrepantes al realizar el test de Grubb's $(p=0,05)$, contraste de dos colas, para este tamaño muestral con un valor crítico de 2,02 . Se obtiene una media aritmética en las 7 series de $61,70 \%$ y $\mathrm{S}$ igual a 0,44 $\%$, que corresponde a la estimación de la desviación estándar de la precisión intermedia o reproductibilidad intralaboratorio. En la tabla 1 se reflejan los datos relativos a la precisión en términos de repetibilidad y precisión intermedia.

El límite de repetibilidad alcanza un valor del 0,58 \% para un nivel de confianza del $95 \%$, y se estima que se encuentra entre $0,40 \%$ y $1,07 \%$ con un nivel de confianza del $95 \%$; y el límite de reproductibilidad, un $1,25 \%$, para un nivel de confianza del 95\%. 
Tabla 1. Resultados correspondientes a la repetibilidad y a la precisión intermedia del procedimiento.

\begin{tabular}{|lccr|}
\hline & S (\%) & DER (\%) & $\mathrm{n}$ \\
\hline Repetibilidad & 0,21 & 0,34 & 10 \\
Reproductibilidad intralaboratorio & 0,44 & 0,71 & 7 \\
\hline
\end{tabular}

S: Desviación estándar. DER (\%): Desviación estándar relativa en \%. n: Número de observaciones.

La precisión intermedia del método o reproductibilidad intralaboratorio $\left(\mathrm{S}_{\mathrm{I}}\right)$, expresada como desviación estándar relativa (DER), alcanza un valor inferior al $1 \%$. La diferencia entre los resultados de dos determinaciones realizadas una después de la otra por el mismo analista debe ser $\leq 0,58 \%$.

Para determinar si el valor medio del contenido de humedad es significativamente diferente del valor certificado, se usa el estadístico t de dos colas, según la expresión (1) del apartado material y métodos. Se obtiene un valor $\mathrm{t}_{\exp }=1,46 ; \mathrm{t}_{\mathrm{tab}}=$ 2,447 para $(n-1)=6, v$, grados de libertad y $\alpha=0,05$. Del mismo modo, el valor certificado cae dentro del intervalo de confianza con una probabilidad del $95 \%$. El sesgo experimental resulta no significativo y se obtiene a partir de la expresión: sesgo $\leq t \alpha / 2$ eff. $u$ (trazabilidad/sesgo), donde $t \alpha / 2$ eff es el valor t tabulado de dos colas para los grados de libertad efectivos, $\mathrm{v}_{\text {eff, }}$ asociados con la incertidumbre (trazabilidad/sesgo).

En cuanto a las muestras duplicadas de una amplia variedad de matrices y concentraciones de analito, se obtiene un valor $\mathrm{S}$ igual a $0,17 \%$, que corresponde a la desviación estándar de las diferencias entre duplicados. Mediante la aplicación de las ecuaciones (2), (3), (4), y (5) del apartado material y métodos, se hallan los valores de incertidumbre correspondientes a duplicados $(0,12 \%)$, precisión $(0,44 \%)$, trazabilidad/ sesgo $(0,39 \%)$ e incertidumbre combinada $(0,60 \%)$. En la Figura 1 se puede comprobar la contribución de la $u_{\text {precisión, }}, u_{\text {tra- }}$ zabilidad/sesgo, $\mathrm{u}_{\text {duplicados, }}$, a la medida de la incertidumbre estándar combinada uc. La incertidumbre expandida U, (1,20\%) se obtiene mediante la expresión (6). Asimismo, se obtiene una relación $\mathrm{u}($ trazabilidad/sesgo $) / \mathrm{u}_{\mathrm{c}}(0,39 / 0,60)$ del $65 \%$. Mediante la aproximación SUMU se añade el sesgo a la incertidumbre expandida, resultando una incertidumbre corregida igual $1,38 \%$.

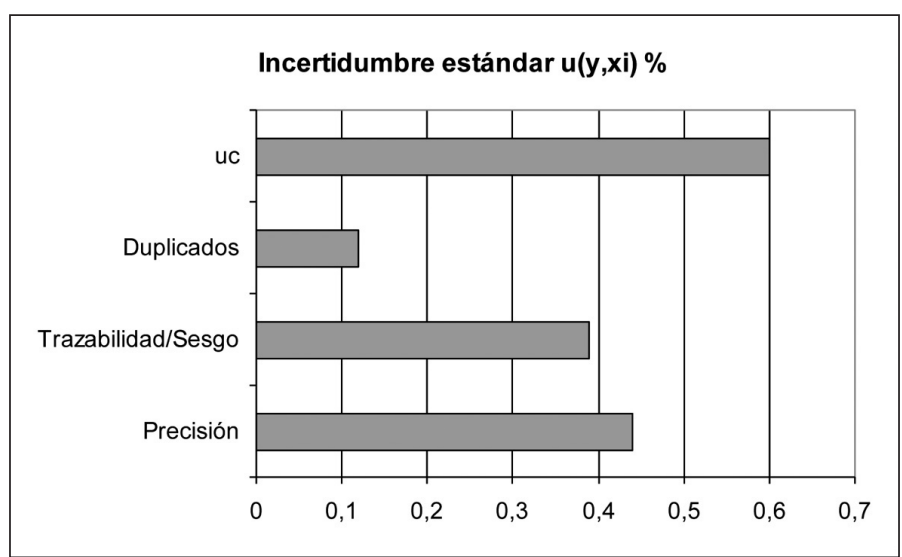

Figura 1. Contribución de la precisión, trazabilidadlsesgo y duplicados a la medida de la incertidumbre estándar combinada, $u_{c}$.

\section{DISCUSIÓN}

La IUPAC ${ }^{22}$ recomienda que la muestra de referencia se analice al menos en 7 réplicas y preferentemente $\geq 10$, en cada nivel de concentración, para obtener una aceptable medida de la exactitud. El criterio más común es realizar 10 réplicas, aunque la ISO 5725-3 recomienda al menos 15. Incluso algunos autores proponen determinar el mínimo número de medidas para evaluar el sesgo ${ }^{23}$. Mediante la adaptación de la norma ISO 5725$6^{24}$ a una situación intralaboratorio ${ }^{25}$, el material de referencia se analiza variando los factores que puedan afectar a los resultados (analista, instrumento, día, replicado), lo que permite estimar la precisión intermedia debida a varios factores, y que corresponde a la varianza asociada a la precisión intermedia de analistas, instrumentos y días diferentes. Asimismo, se considera aceptable la diferencia máxima del $0,58 \%$ entre los resultados de dos determinaciones realizadas una después de la otra por el mismo analista. El hecho de no hallar resultados discrepantes indica que el método es preciso y exacto, y el proceso de aseguramiento de la calidad se encuentra bajo control.

Según el teorema del límite central ${ }^{26}$, los resultados obtenidos de medidas repetidas se distribuyen generalmente de acuerdo a curvas gaussianas, lo que permite aplicar inferencias de estadística paramétrica.

Es interesante señalar que el contenido de humedad de los platos preparados se ajusta al intervalo 40-95\%. Por ello, se verifica la trazabilidad en un intervalo restringido de concentraciones, y por tanto, se asume que el sesgo es igual en todo el intervalo de concentraciones. Hay que tener presente que en el análisis nutricional llegar a través de una cadena ininterrumpida de comparaciones a la unidad de materia del SI de unidades no siempre es factible ${ }^{27}$. Una justificación posible es que en la determinación de humedad la pérdida no solamente es de agua, sino que también pueden existir ligeras pérdidas de componentes volátiles del alimento. En metrología física el SI de unidades es el punto final de trazabilidad y en análisis químico se mantiene la posición de que la mayoría de medidas no son trazables al mol. Hay que tener en cuenta que la trazabilidad en el análisis de macronutrientes es compleja, más propensa a errores debido a los múltiples pasos en las operaciones preliminares ${ }^{28}$. Sería imposible conseguir, por ejemplo, una trazabilidad al mol en la fase de extracción de la grasa e incluso el resultado de la determinación de humedad debido a que, la extracción o preparación de la muestra, constituye un eslabón débil en la cadena de trazabilidad y la trazabilidad del resultado tiene su propio valor límite ${ }^{29}$. Otro aspecto importante en el análisis nutricional es que los macronutrientes (grasa, fibra, etc.) poseen una naturaleza química mal definida. Por ejemplo, la grasa es un analito empírico que consiste en una mezcla de triglicéridos, ácidos grasos, esteroles, etc. Por consiguiente, no podríamos trazar un análisis a grasa pura, a causa de que la grasa pura podría tener un rango de composiciones ${ }^{30}$. Del mismo modo, como es imposible aislar un mol de un macronutriente puro, explica la complejidad para establecer la trazabilidad de un resultado. Por consiguiente, para verificar la trazabilidad se utiliza un material de referencia certificado (MRC), con un nivel de trazabilidad más bajo que otras referencias, comoquiera que sus resultados no se han obtenido con métodos primarios; pero 
desde un punto de vista práctico constituyen la referencia más adecuada para verificar la trazabilidad en análisis de macronutrientes. Otro factor a tener en cuenta es que el sesgo también parece verse afectado por la complejidad de las matrices, según se desprende de un estudio sobre la determinación de humedad en diferentes matrices complejas orgánicas e inorgánicas utilizando el método de secado en horno, donde se observa un sesgo variable en relación al valor de referencia ${ }^{31}$. Por tanto, la evaluación y cuantificación del sesgo y su magnitud, se incluye como un componente de incertidumbre ${ }^{32}$.

El análisis por duplicado de diversas matrices de platos preparados correspondientes a una amplia variedad de muestras, permite estimar la incertidumbre de duplicados que incluye variabilidad natural de aquellos factores que afecten a los resultados.

La evaluación de la exactitud utilizando muestras adicionadas representativas de las matrices de rutina con una cantidad conocida de analito también es utilizada por los laboratorios. Esta referencia tiene un nivel de trazabilidad más bajo que el $\mathrm{MRC}$, debido a que el analito añadido podría no reproducir el comportamiento real del analito presente en la muestra.

Es evidente que cuando se concluye erróneamente que el sesgo del método no es significativo, la incertidumbre de los resultados puede estar subestimada, y esta subestimación será tanto mayor cuanto mayor sea la contribución de la incertidumbre del sesgo a la incertidumbre combinada. Cuando la contribución del sesgo es mayor del $30 \%$ de la incertidumbre de los resultados puede estar muy subestimada ${ }^{33}$. En nuestro caso la incertidumbre del sesgo es elevada, ya que se parte de una referencia que tiene una incertidumbre expandida del 0,7\%. Una solución plausible es analizar la referencia más veces para reducir la incertidumbre del sesgo y la probabilidad de un error de $2^{\text {a }}$ especie o error $\beta$, al concluir que el procedimiento es trazable cuando en realidad no lo es. Por ejemplo, para un valor de $\Delta$ igual a 1 y $\alpha=0,05$, y para reducir la probabilidad de un $1 \%$ a un $5 \%$ de cometer un error $\beta$ se debería aumentar el número de replicas de 13 a $18^{34}$.

En realidad, siempre habrá una diferencia entre el valor medio obtenido al analizar el material certificado de referencia y el valor certificado debido a errores aleatorios y también a un posible error sistemático. Al realizar la prueba de significación se obtiene una $t_{\text {exp }}$ menor que la $t_{\text {tab }}$, lo que confirma una buena exactitud, ya que se verifica si esa diferencia es únicamente debida a errores aleatorios y errores sistemáticos no significativos $\mathrm{y}$, por tanto, se concluye que el método no produce resultados sesgados o errores sistemáticos significativos, es trazable al valor certificado, y se puede trasladar la trazabilidad a muestras futuras. En un ejercicio interlaboratorio sobre determinación de humedad en un producto cárnico, se obtiene un z zcore de 0,16 , lo que indica que las variables que afectan a los resultados están muy bien controladas. Aunque los tests estadísticos nos indiquen que los resultados sean trazables nunca se tendrá una total seguridad en esta afirmación. La aplicación de estos tests puede llevar consigo el riesgo de cometer un error $\alpha$ o de primera especie, al concluir que el procedimiento no es trazable cuando en realidad lo es; $y$ un error de $2^{\mathrm{a}}$ especie o error $B$.

La estrategia general para la estimación de la incertidumbre propuesta por la ISO $^{35}$, también conocida como aproximación "bottom-up", recogida en diversas publicaciones ${ }^{36-38}$, hace que el proceso sea mucho más trasparente; pero más laborioso. Consiste en identificar componentes individuales de incertidumbre o fuentes de incertidumbre del mensurando, que en este caso afecta a la masa de la muestra antes y después de la desecación, que depende de la precisión y calibración de la balanza, con parámetros asociados de linealidad, repetibilidad y resolución digital. Además, existen otras fuentes de incertidumbre como la pureza del reactivo, temperatura de desecación, tiempo de desecación, volumen del reactivo añadido, un factor de heterogeneidad debida a la trituración durante la preparación de la muestra ${ }^{39,40}$, variabilidad de las muestras y eliminación de partes no comestibles, por lo que el cálculo de cada una de ellas resulta complejo; en consecuencia, y debido a la complejidad de estimar correctamente cada una de las fuentes de incertidumbre significativas, se considera el proceso analítico de forma global incluyendo la variabilidad de las muestras por su diversidad y por su heterogeneidad. La utilización de un diagrama causa y efecto constituye una herramienta de ayuda al analista a identificar de forma estructurada las fuentes de incertidumbre y cuantificar cada una de ellas ${ }^{41-44}$. Con la estrategia ISO se cuantifica cada componente a través del proceso experimental, a través de datos bibliográficos, y se realiza su estimación y se combinan todas las fuentes de incertidumbre para dar lugar a la incertidumbre total. Esto hace que sea difícil calcular la incertidumbre de una forma correcta con esta estrategia. En este trabajo se realiza el cálculo de la incertidumbre de una forma global, considerando el proceso analítico de forma conjunta, y siguiendo las recomendaciones de la guía EURACHEM/CITAC $2000^{45}$ y de otros autores ${ }^{46}$, se aprovecha la validación del método para calcular la incertidumbre de los resultados, de tal manera que en este caso se agrupan los términos incertidumbre de la precisión, incertidumbre de la trazabilidad/sesgo e incertidumbre de duplicados, lo que permite realizar el cálculo de una forma global.

La presencia de otros términos de incertidumbre, como es el caso de la incertidumbre de duplicados, ejerce un efecto favorecedor al producir una disminución de la incertidumbre del sesgo en la incertidumbre final. Y cuanto mayor sea la incertidumbre de otros términos, menos se subestima la incertidumbre de los resultados.

Con esta aproximación el esfuerzo requerido para estimar las fuentes de incertidumbre no consideradas en la validación del procedimiento (heterogeneidad de la muestra, efecto matriz, etc.) es considerablemente menor que el esfuerzo necesario para estimar la incertidumbre con la aproximación ISO. Por consiguiente, los principales beneficios de esta aproximación son su conceptualidad y su simplicidad práctica. En el análisis de macronutrientes existen una serie de inconvenientes, la mayor parte de los cuales son debidos al limitado número de $\mathrm{MRC}$, en cuanto a matrices y concentraciones de analito.

Es interesante señalar que la relación entre la incertidumbre del sesgo y la incertidumbre combinada es elevada; por ello, se decide que el sesgo no significativo se incluya como un componente de la incertidumbre para evitar una infraestimación de la incertidumbre de los resultados. Cuánto más alta sea esta contribución más importante es incluir el sesgo experimental, de ahí que se haya utilizado el método SUMU ${ }^{47}$ que añade el sesgo a la incertidumbre expandida. 


\section{CONCLUSIONES}

- El uso de un diseño anidado de 4 factores (analista, instrumento, día, replicado) permite variar los factores de una forma ordenada entre cada serie, y estimar la precisión intermedia debida a varios factores.

- El cálculo de la incertidumbre de forma global aprovechando el proceso de validación del procedimiento supone una ventaja en cuanto a simplicidad, especialmente para laboratorios con pocos recursos humanos y materiales.

- La inclusión del sesgo experimental no significativo como un componente de la incertidumbre tiende a evitar la infraestimación de la incertidumbre de los resultados.

\section{BIBLIOGRAFÍA}

1. UNE-EN ISO/IEC 17025. Requisitos generales relativos a la competencia de los laboratorios de ensayo y calibración. AENOR, Madrid. 2000.

2. Vocabulario científico y técnico. Real Academia de Ciencias Exactas, Físicas y Naturales. Espasa. Madrid 1996.

3. Bonet-Domingo E, Escuder-Gilabert L, Medina-Hernández MJ, Sagrado S Uncertainty-Based Internal Quality Control. Harmonization considerations. Anal Chem 2006; 78:8113-8120.

4. Thompson M, Fearn T. What Exactly is Fitness for Purpose in Analytical Measurement? Analyst 1996; 121:275-278.

5. International Vocabulary of Basic and General Terms in Metrology (VIM), ISO, Gèneva, Switzerland, 1993.

6. Thompson M. Comparability and traceability in analytical measurements and reference materials. Analyst 1997; 122:1201-1205.

7. Barwick VJ, Ellison SLR, Rafferty MJQ, Rattanjit SG. The evaluation of measurement uncertainty from method validation studies. Part 2: The practical application of laboratory protocol. Accred Qual Assur 2000; 5: 104-113.

8. Thompson M, Ellison S.L.R., Wood R.A. Harmonised Guidelines for singlelaboratory validation of methods of analysis. Pure Appl Chem 2002; 74 (5): $835-855$.

9. ISO 3534-1. Statistics, Vocabulary and symbols. Genève. 1993.

10. Thompson M. Analyst 1997; 122: 1201-1206.

11. Maroto A, Boqué R, Riu J, Rius X. Evaluating uncertainty in routine analysis. Trends in Analytical Chemistry. 1999; 18:577-584.

12. De Melo Abreu, S, Caboni P, Cabras P, Garau VL, Alves A. Validation and global uncertainty of a liquid chromatographic with diode array detection method for the screening of azoxystrobin, kresosim-methyl, trifloxystrobin, famoxadone, pyraclostrobin and fenemidone in grapes and wine. Analytica Chimica Acta. 2006; 573-574:291-297.

13. Serra R, Mendoça C, Abrunhosa L, Pietri A, Venâncio A. Determination of ochratoxin A in wine grapes: comparison of extraction procedures and method validation. Analytica Chimica Acta 2004; 513:41-47.

14. Cowles JR, Daily S, Ellison SLR, Hardecastle W, Williams C. Experimental sensitivity analysis applied to sample preparation uncertainties: are ruggedness tests enough for measurement uncertainty estimates? Accred Qual Assur 2001; 6:368-371.

15. Norma ISO 1442: 1997. Meat and meat products. Determination of moisture content.

16. ISO Guide 5725-3:1994/Cor.1:2001. Accuracy (Trueness and Precision) of Measurement Methods and Results. Part 3: Intermediate measures of the precision of a standard measurement method. Genève.

17. Lyn J A, Ramsey MH, Fusell RJ, Wood R. Measurement uncertainty from physical sample preparation: estimation including systematic error. Analyst 2003; 128:1391-1396.

18. Boqué R, Maroto A, Riu J, Rius X. Validation of analytical methods. Grasas y Aceites 2002; 53 (1): 128-143.

19. BIPM, CEI, IFCC, ILAC, ISO, UICPA, UIPPA, OIML. Evaluation of measurement data-Guide to the expression of uncertainty in measurement. First Edition 2008.
20. Bettencourt da Silva RJN, Santos JR,Camoes GFC. Worst case uncertainty estimates for routine instrumental analysis. Analyst 2002; 127: 957-963.

21. Maroto A, Boqué R, Riu J, Rius X. Effect of non-significant proportional bias in the final measurement uncertainty. Analyst 2003; 128:373-378.

22. Fajgelj A, Ambras A. Project 5/2/99. Harmonised Guidelines for In-House Validation of Methods of Analysis. International Union of Pure and Applied Chemistry. IUPAC. 1999.

23. Kuttatharmmakul S, Massart DL, Smeyers-Verbeke J. Comparison of alternative measurement method: determination of the minimal number of measurements required for the evaluation of the by means of interval hypothesis testing. Chemometrics and intelligent laboratory systems. 2000; 52:61-73.

24. ISO Guide 5725-6:1994/Cor.1:2001. Accuracy (Trueness and Precision) of Measurement Methods and Results. Part 3: Intermediate measures of the precision of a standard measurement method. Genève.

25. Kuttatharmmakul S, Massart DL, Smeyers-Verbeke J. Comparison of alternative measurement methods. Analytica Chimica Acta. 1999; 391:203-225.

26. Webster A. Estadística aplicada a los negocios y a la economía. Colombia: McGraw-Hill, $3^{\mathrm{a}}$ Edición 2000: 142-167.

27. King B. Traceability of Chemical Analysis.Analyst 1997; 122: 197-204.

28. Valcárcel M, Ríos A. Traceability in Analytical Chemistry. Analyst 1995; 2291-2297.

29. King B. Metrology and analytical chemistry bridging the cultural gap. Metrologia 1997; 34:41-47.

30. Vogl J, Ostermann M. On the measurement of the moisture content in different matrix materials. Accred Qual Assur 2006; 11: 356-362.

31. Thompson M. Sense and traceability. Analyst 1996; 121:285-288.

32. Kane JS. Analytical Bias: the Neglected Component of Measurement Uncertainty. Analyst 1997; 122: 1283-1288.

33. Maroto A, Boqué R, Riu J, Rius X. Should non-significant bias be included in the uncertainty budget?. Accred and Qual Assur 2002; 7: 90-94.

34. Milton JS. Estadística para la biología y ciencias de la salud. Madrid: McGraw-Hill Interamericana. $3^{\text {a }}$ Edición 2001; 512-542.

35. BIPM, IEC, IFCC, ISO, IUPAC, IUPAP, OIML. Guide to the expression of uncertainty in measurement ISO, Genève, 1993.

36. Analytical Methods Committee. Uncertainty of Measurement: Implications of its use in Analytical Science. Analyst 1995; 120:2303-2308.

37. Stepan R, Hajslova J, Kocourek V, Ticha J. Uncertainty of gas chromatographic measurement of troubles pesticide residues in apples employing conventional and mass spectrometric detectors. Analytica Chimica Acta 2004; 520: 245-255.

38. Yenisoy-Karakas S. Validation and uncertainty assessment of rapid extraction and clean-up methods for the determination of 16 organochlorine pesticides residues in vegetables. Analytica Chimica Acta 2006; 571: 298-307.

39. Ferrus R, Torrades F. Bias-free adjustement of analytical methods to laboratory samples in routine analytical procedures. Anal Chem 1988; 60:12811285.

40. Lyn JA, Ramsey MH, Wood R. Optimised uncertainty in food analysis: application and comparison between four contrasting "analyte-commodity" combinations. Analyst 2002; 127:1252-1260.

41. Lisinger TPJ, Führer M, Kander W, Schuhmacher R. Determination of measurement uncertainty for the determination of triazines in ground water from validation data. Analyst 2001; 126:211-216.

42. Brix R, Hansen HS, Barwick V, Tjornelund J. Method optimisation with the use of uncertainty budgets. Analyst 2002; 127:140-143.

43. Populaire S, Campos Giménez E. A simplified approach to the estimation of analytical measurement uncertainty. Accred Qual Assur 2006; 10:485-493.

44. Ellison SLR, Barwick VJ. Using validation data for ISO measurement uncertainty estimation. Part 1 . Principles of an approach using cause and effect analysis. Analyst 1998; 123: 1387-1392.

45. Ellison SLR, Rosslein M, Williams A. EURACHEM/CITAC Guide. Quantifying Uncertainty in Analytical Measurement. $2^{\mathrm{a}}$ Edition. 2000.

46. Bettencourt da Silva RJN, Lino JM, Santos JR, Camoes FGFC. Estimation of precision and efficiency mass transfer steps for determination of pesticides in vegetables aiming at the expression of results with reliable uncertainty. Analyst 2000; 125: 1459-1464.

47. Synek V. Effect of significant bias and its uncertainty on the coverage probability or uncertainty intervals. Part 1 . Evaluation for given value of true bias. Talanta 2006; 70: 1024-1034. 